\title{
A PROPOS DE LA DYSTROPHIE MYOTONIQUE
}

\author{
Y. DesnoYers, M.D., F.R.C.P.(c) ${ }^{\circ}$
}

LA DYSTROPHE myotonique est une maladie héréditaire, d'allure systémique, et présente de la deuxième à la quatrième décade de la vie. La maladie est susceptible de créer une multitude de problèmes en anesthésie, à cause de l'insuffisance fonctionnelle de plusieurs organes. La physiopathologie a ici fait l'objet d'hypothèses multiples ${ }^{1}$ et à la lumière d'un cas clinique, nous tenterons d'y ajouter un nouvel aspect.

\section{SYMPTOMES}

Les malades présentent une atrophie musculaire fréquemment disséminée, affectant en particulier les muscles de la respiration (diaphragme, muscles intercostaux, musculature abdominale, sternocleidomastoidien, larynx), également les muscles de la face, ceux des membres, le trapèze. Le syndrome myotonique est caractéristique (persistance de la contraction musculaire après cessation d'un effort volontaire). La maladie se caractérise également par l'incidence accrue de cataractes, calvitie frontale, atrophie gonadique, insuffisance cortico-surrénalienne, hypométabolisme, troubles de conduction myocardique, perturbations du métabolisme du glucose, ${ }^{1-3}$ atteinte mentale.,

\section{PHYSTOPATHOLOGIE}

La maladie se caractérise par une atteinte musculaire (Denny-Brown, 1941), avec anomalie de l'excitabilité de la fibre musculaire qui fait qu'une excitation conduit à la décharge répétée d'un courant d'action dans la fibre musculaire, effet non réductible par les curares non dépolarisants. Au point de vue histologique, l'atteinte de la fibre musculaire est caractéristique: $(a)$ noyaux placés, sous le sarcolemne, en rangées centrales dans des fibres musculaires par ailleurs normales; $(b)$ plaques terminales petites mais allongées; $(c)$ réseau de fibres nerveuses se ramifiant autour de fibres musculaires ou pénétrant dans la fibre; $(d)$ ramification terminale très profuse des fibres nerveuses avec formation de multiples plaques terminales sur la même fibre musculaire.,

La symptomatologie musculaire pourrait théoriquement s'expliquer soit par un excès d'acétyl choline produit à la terminaison nerveuse ${ }^{8}$ (Dundee, 1951), ou par une diminution d'activité des cholinestérases. Il serait plus probable, cependant, que les fibres musculaires soient anormalement sensibles à l'acétyl choline ${ }^{1}$ et que l'accommodation de la fibre musculaire soit déficiente, ${ }^{7}$ avec possibilité d'un trouble métabolique intra-cellulaire permettant au potassium de sortir plus facilement de la cellule.

'Départment d'anesthésie, Hôpital Ste-Jeanne d'Arc, Montréal, Québec. 


\section{DANGERS De L'ANESTHESIE}

La première description de risque anesthésique dans la dystrophie myotonique est due à Johnson et Marshall en 1915, qui rapportent un cas de dystrophie myotonique, cause, sous anesthésie à l'éther, d'une insuffisance respiratoire avec cyanose qui dura 24 heures. Par la suite, on rapporta dans cette maladie des cas d'apnée prolongée durant de 30 à 45 minutes et de relâchement musculaire excessif $^{6,8}$ à la suite de faibles doses de thiopentone $(100 \mathrm{mg}$ ); on crut à une action périphérique et non pas centrale du thiopentone. Des cas semblables furent rapportés par Bourke (1957) et Pachomov (1958); cependant, dans tous ces cas, Kaufman ${ }^{1}$ met en doute l'effet spécifique du médicament, ces patients ayant tous reçu également une multitude de drogues susceptibles de déprimer la respiration (péthidine, morphine, succinylcholine, etc.) et il suggère plutôt un effet additif de dépression centrale chez des patients ayant déjà une réserve respiratoire diminuée.

Ces malades présentent également un risque exagéré du point de vue pulmonaire. L'incidence d'infection pulmonaire postoperatoire est accrue, ${ }^{2}$ l'hypoventilation pré et surtout, postopératoire est fréquente et exagérée par les médicaments dépresseurs de la respiration. ${ }^{3,8,9}$ Ces malades sont davantage susceptibles à la pneumonie d'aspiration par insuffisance des mécanismes protecteurs lors de la déglutition. ${ }^{10}$ On a rapporté également la possibilité de myotonie des muscles respiratoires conduisant d l'insuffisance respiratoire (Kaufman, 1960), dont un cas à la suite d'injection de succinylcholine. ${ }^{11}$

Le système cardiovasculaire peut également être perturbé principalement sous forme de troubles de conduction variés, et amener des perturbations secondaires au cours de l'anesthésie.

Enfin, mentionnons une sensibilité accrue à tous les médicaments, en particulier sous anesthésie, due à un hypométabolisme et des dangers secondaires à l'insuffisance cortico-surrénalienne parfois présente.

\section{Presentation d'un Cas}

M. A.R., 21 ans, 106 livres, est opéré d'urgence pour appendicite suppurée et péritonite secondaire. Le patient est très souffrant et le questionnaire préopératoire est difficile. Le dossier actuel ne mentionne aucune pathologie antérieure. Le malade reçoit en prémédication: Mépéridine, $75 \mathrm{mg}$ et atropine $0.4 \mathrm{mg}$. L'induction est faite avec $325 \mathrm{mg}$ de thiopental, puis après quelques minutes, il est possible d'assister la respiration au masque en circuit semi-fermé, avec $\mathrm{N}_{2} \mathrm{O}$ à $4 \mathrm{~L} / \mathrm{min}, \mathrm{O}_{2}$ à $2 \mathrm{~L} / \mathrm{min}$ et halothane de 0.5 à 1 pour cent. Le malade n'est pas intubé. A l'ouverture du péritoine, $30 \mathrm{mg}$ de succinylcholine est injecté intraveneusement pour obtenir le relâchement musculaire, et le malade tombe en apnée. La même dose est répétée après une autre 10 minutes, pour augmenter le relâchement musculaire quoique le malade soit encore apnéique à ce moment. Le malade demeure en apnée à la fin de l'intervention, il est intubé facilement, et il s'écoule 60 minutes entre la première injection de succinylcholine et le retour 
à la respiration spontanée. La revision subséquente du dossier antérieur du patient nous fait découvrir qu’il souffrait de "dystrophie myotonique" prouvée depuis deux ans.

\section{INVESTIGATIONS}

Par la suite, pour éprouver la susceptibilité du malade au thiopental et investiguer ses pseudocholinestérases, nous avons fait les épreuves suivantes:

1. Nous avons injecté au patient, non prémédiqué, $200 \mathrm{mg}$ de thiopental à 2.5 pour cent, intraveineusement. Il s'enuivit une période d'apnée d'environ 45 secondes, puis le patient s'éveilla alerte et sans dépression respiratoire, après deux à trois minutes, manifestant ainsi une réaction tout à fait normale.

\section{TABLEAU I}

Etude des Pseudocholinestérases du Patient

\begin{tabular}{llll}
\hline \hline $\begin{array}{l}\text { Dosage } \\
\text { (N: } 40 \text { à } 80 \text { unités) }\end{array}$ & $\begin{array}{l}\text { Activité cholinestérasique } \\
\text { (N: } 126 \text { à } 190 \text { unités) }\end{array}$ & $\begin{array}{l}\text { "Dibucaine } \\
\text { number" } \\
\text { (N: 71 unités) }\end{array}$ & $\begin{array}{l}\text { "Fluoride } \\
\text { number" } \\
\text { (N: } 60 \text { unités) }\end{array}$ \\
\hline 15.2 & 118 & 18 & 24 \\
\hline
\end{tabular}

2. Le Tableau $\mathrm{I}^{12}$ est compatible avec des pseudocholinestérases atypiques du type homozygote. ${ }^{13}$

Nous avons investigué subséquemment les pseudocholinestérases plasmatiques de la sœur du patient (A), qui souffre également de dystrophie myotonique, du père (B) et de la mère (C) qui, eux, sont asymptomatiques. Nous avons obtenu les résultats que montre le Tableau II.

TABLEAU II

\begin{tabular}{llll}
\multicolumn{2}{c}{ Etude des Pseudocholinestérases des } & Autres Membres de la Famille \\
\hline \hline Sujet & Activité cholinestérasique & "Dibucaine & "Fluoride \\
number" & number" \\
\hline A & 177 & 63 & 41 \\
B & 214 & 60 & 42 \\
c & 224 & 64 & 46 \\
\hline
\end{tabular}

Les résultats du Tableau II sont tous compatibles avec des pseudocholinestérases atypiques, mais du type intermédiaire ou heterozygote (cas peu sensibles à la succinylcholine).

\section{Discussion}

La physiopathologie de la dystrophie myotonique a fait l'objet de plusieurs hypothèses dans le passé, les unes s'appuyant sur une déficience du métabolisme ou de la structure de la fibre musculaire ${ }^{14}$ elle-même (Lanari, 1946; Landau, 1952; Kaufman, 1959) pour expliquer le phénomène myotonique, les autres mentionnant la possibilité de pathologie de la jonction myo-neurale ${ }^{8,16}$ pour expliquer la dystrophie. Quoique la possibilité de mécanismes cholinestérasiques soit men- 
tionnée, nous n'avons découvert aucun autre rapport de cas d'atypie pseudocholinestérasique du type homozygote, prouvé, dans cette maladie. ${ }^{15}$ La sour, qui souffre de la même maladie, le père et la mère du patient possèdent tous un gène atypique et un gène normal dans leurs cholinestérases. Nous nous questionnons donc sur la possibilité qu'il y ait relation, dans certains cas de dystrophie myotonique, entre un tel gène et la maladie.

Il demeure que l'atypie pseudocholinestérasique homozygote pourrait parfois être un facteur associé à maladie, et pourrait expliquer un certain nombre de cas d'apnée prolongée ou de dépression respiratoire mentionnés par plusieurs auteurs à la suite d’anesthésies chez ces patients.

L'étude des pseudocholinestérases devrait, à notre avis, faire partie de l'investigation de tout cas de dystrophie myotonique, en particulier en prévision d'une anesthésie, où une atypie constituerait une contre-indication de plus à l'utilisation de curarisants du type dépolarisant dans ces cas.

\section{RÉSUMÉ}

La dystrophie myotonique est une maladie héréditaire impliquant une multitude de systèmes, accompagnée de myotonie et d'atrophie musculaire. L'histologie de la fibre musculaire et celle des plaques terminales est caractéristique. La physiopathologie fait l'objet de théories multiples impliquant des perturbations du métabolisme de la myofibrille ou de la jonction myoneurale. L'anesthésie de ces patients présente des problèmes reliés à une sensibilité accrue aux dépresseurs de la respiration. Nous avons présenté la première description d'atypie pseudocholinestérasique homozygote dans un cas de dystrophie myotonique. Après investigation familiale, nous croyons ajouter une nouvelle hypothèse génétique possiblement reliée à certains de ces cas ou du moins mentionner un nouveau danger relié à l'anesthésie de ces patients.

\section{SUMMARY}

Dystrophia myotonica is hereditary and causes systemic effects subsequent to myotonia and muscular atrophy. Pathohistological findings in the muscle fibre and the terminal plate are characteristic. Physiopathological theories are numerous and are related to metabolic disturbances of either the muscle or the neuromuscular junction. These patients are hypersensitive to respiratory depressants used during anaesthesia. A new description is presented of atypical heterozygotous pseudocholinesterases found in a case of dystrophia myotonica. Following familial investigation, a new genetic hypothesis is formulated in an attempt to explain another danger in relation to anaesthesia in these patients.

\section{REMERCIEMENTS}

Au Dr. Nancy E. Simpson du Queen's University, Kingston, Ontario pour avoir fait gracieusement les études des pseudocholinestérases, au Dr. N. Bouziane, chef de notre laboratoire, pour sa collaboration et aux membres réguliers de notre service d'anesthésie pour leurs encouragements. 


\section{REFERENCES}

1. Kaufman, L. Anaesthesia in Dystrophia Myotonica. Proc. Roy. Soc. Med. 53: 183 (1959)

2. Gillam, P. M.; Heaf, P. J.; \& Kaufman, L. Respiration in Dystrophia Myotonica Thorax. 19: 112 (1964).

3. LEe, F. I. \& Hughes, D. T. Systemic Effects in Dystrophia Myotonica. Brain. 87: 521 (1964).

4. MACDERmot, V. The Histology of the Neuromuscular Junction in Dystrophia Myotonica. Brain. 84: 75 (1961).

5. Rosman, N. P. \& Reberz, J. J. The Cerebral Defect and Myopathy in Myotonic Dystrophy. Neurology. 17: 1106 (1967).

6. DundeE, J. W. Thiopentone in Dystrophia Myotonica. Curr. Res. Anaesth. $31: 257$ (1952).

7. Geschwind, N. \& Simpson, J. A. Procaine Amide in the Treatment of Myotonia. Brain. 78: 81 (1955).

8. MCClelland, R. M. The Myasthenic State and the Myotonic Syndrome. Brit. J. Anaesth. 32: 81 (1960).

9. Welsh, J. D.; HaAse, G. R.; \& Bynum, T. E. Myotonic Muscular Dystrophy. Arch. Internat. Med. 114: 669 (1964).

10. Pruzanski, W. \& Profis, A. Pulmonary Disease in Myotonic Dystrophy. Am. Rev. Resp. Dis. 91: 874 (1965).

I1. Paterson, I. S. Generalized Myotonia Following Suxamethonium. Brit. J. Anaesth. 34: 340 (1962).

12. Brodeur, J.: Martin, P.; Authier, L.; \& Leber, E. Pseudocholinestérase atypique et apnée prolongée à la succinylcholine. Union Med. 97: 1025 (1968).

13. Wrime \& Churchiml-Davimson. A Practice of Anaesthesia, 2nd ed. Lloyd-Luke Ltd. (1966).

14. Brown, G. L. \& Harvey, A. M. Congenital Myotonia in the Goat. Brain. 62: 341 (1939).

15. Pearson, C. M. Serum Enzymes in Muscular Dystrophy. New England J. Med. 256: 1069 (1957).

16. BraIN, Russel. Diseases of the Nervous System, 5th ed. London: Oxford University Press (1960). 